\title{
Skip metastasis to portocaval and retropancreatic space in T2 cecal cancer without regional and principle lymph node involvement
}

\author{
Soomin Nam', Yoon Jung Choi ${ }^{2}$, Youngki Hong', Jung Gu Kang' \\ Departments of ${ }^{1}$ Surgery and ${ }^{2}$ Pathology, National Health Insurance Service Ilsan Hospital, Goyang, Korea
}

\begin{abstract}
Lymph node metastasis in early colon cancer is relatively low. Furthermore, skip metastasis beyond principle lymph node is very rare. This is a case of early cecal cancer with skip metastasis to portocaval and retropancreatic space, without regional lymph node metastasis. A 69-yearold female diagnosed as cecal adenocarcinoma. The imaging study revealed as early cecal cancer without enlargement of regional lymph node. However, there is enlargement of portocaval lymph node and high fluorodeoxyglucose (FDG) uptake in positron emission tomography scan image. Right hemicolectomy with extended lymph node dissection was done including retropancreatic, portocaval and hepatoduodenal ligament lymph node. Though whole abdominal cavity exploration was done, there was no evidence of other synchronous cancer. The final pathologic findings revealed the poorly differentiated adenocarcinoma with invasion of submucosal layer and focally superficial layer of muscularis propria. Two of total 27 lymph nodes were involved by metastastatic adenocarcinoma which were from portocaval and retropancreatic space. The detailed preoperative imaging study could find unexpected lymph node metastasis beyond range of routine lymph node dissection. Even though the preoperative clinical stage is relatively early, the detailed and sufficient evaluation for clinical and imaging findings is important not to ignore skip metastasis.
\end{abstract}

Keywords: Cecal neoplasms, Colon neoplasms, Lymph node excision, Lymphatic metastasis, Skip metastasis

\section{INTRODUCTION}

In colon cancer, radical surgery including lymph node dissection up to the origin of the feeding artery is commonly considered as a standard procedure. This principle is based on the sequential lymphatic spread of colon cancer. In cases of early colon cancer, the extent of lymph node dissection can be decreased because early colon cancer including T1 or T2 has a $0 \%$ to $20 \%$ chance of lymph node metastasis [1-3]. Therefore, the optimal extent of lymph node

Received: Oct 19, 2017 Accepted: May 21, 2018

Correspondence to: Jung Gu Kang

Department of Surgery, National Health Insurance Service Ilsan Hospital, 100 Ilsan-ro, Ilsandong-gu, Goyang 10444, Korea

Tel: +82-31-900-0001, Fax: +82-31-900-0343

E-mail: kangski@nhimc.or.kr

*This research was presented as a poster presentation at 50th Golden Anniversary congress of KSCP (Korean Society of Coloproctology) from March 29 to April 2, 2017 in Seoul, Korea.

Copyright ( $($ Korean Society of Surgical Oncology

This is an Open Access article distributed under the terms of the Creative Commons Attribution Non-Commercial License (http://creativecommons.org/licenses/by-nc/4.o) which permits unrestricted non-commercial use, distribution, and reproduction in any medium, provided the original work is properly cited. dissection depends on the clinical stage of colorectal cancer.

However, there are some reports about skip lymph node metastasis in which distant lymph nodes are positive but regional lymph nodes are negative for cancer involvement. Skip metastasis occurs in $1 \%$ to $3 \%$ of colon cancer [4]. This is a report about a very rare case of colon cancer that has no regional or principle lymph node involvement but has distant lymph node metastasis such as retropancreatic and portocaval lymph node. Written informed consents were obtained.

\section{CASE REPORT}

A 69-year-old female with a history of hypertension and diabetes mellitus came to the outpatient clinic due to positive results of a stool occult blood test during a routine health examination. Colonoscopic exam revealed a large villous polyp of about $3 \mathrm{~cm}$ in the cecum (Fig. 1). Gross features of the polyp were that of a benign polyp but biopsy results revealed an adenocarcinoma. The level of carcinoembryonic antigen was $2.4 \mathrm{ng} / \mathrm{mL}$, which is within normal limits.

She was referred to the department of general surgery for opera- 


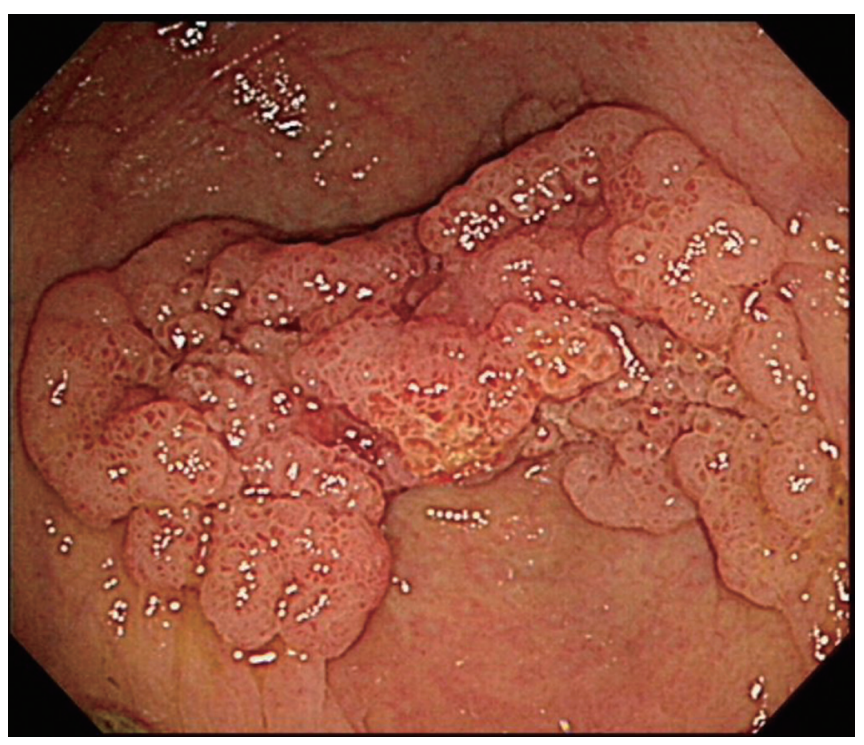

Fig. 1. Colonoscopic finding of cecal villous polyp.
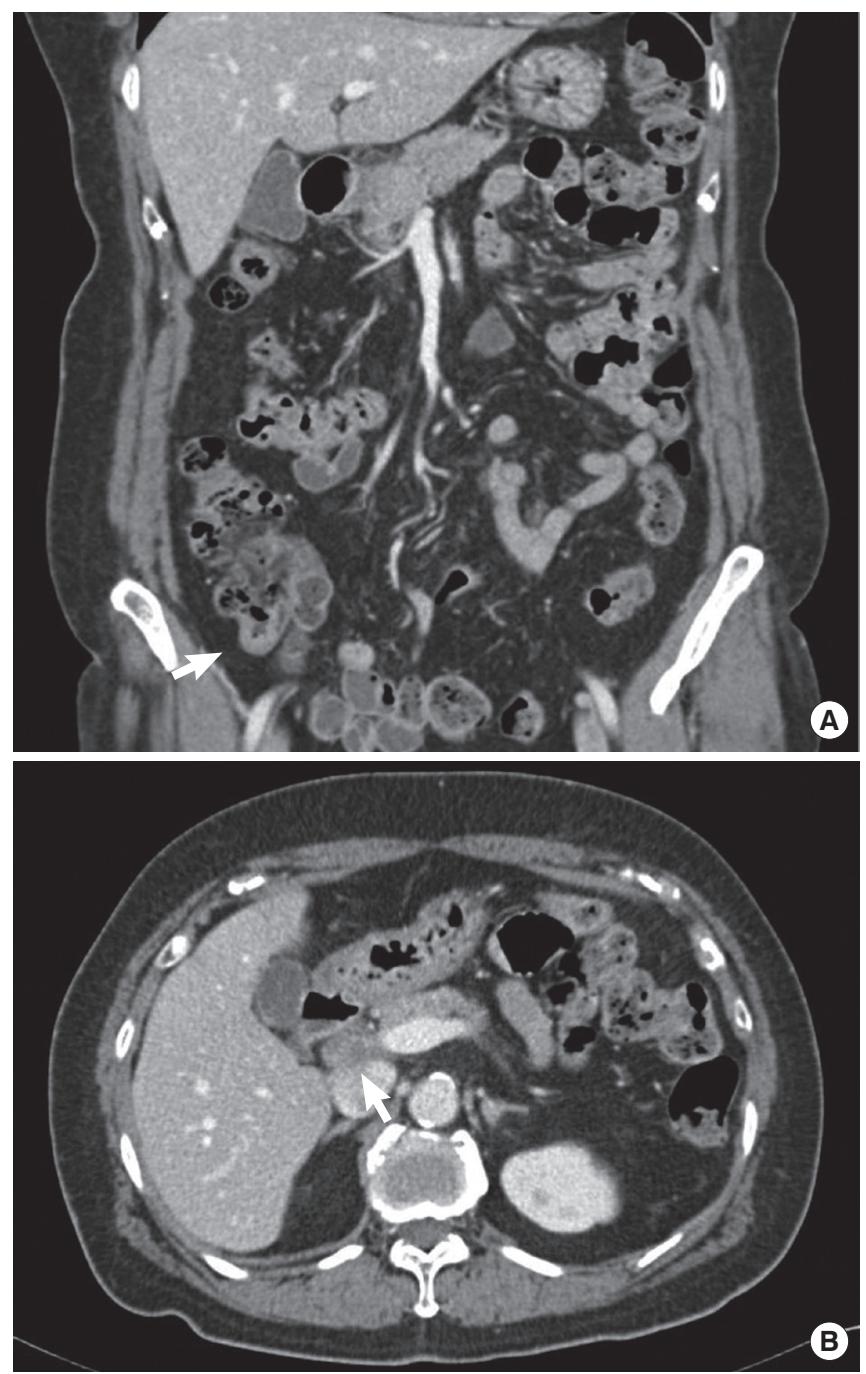

Fig. 2. Abdominopelvic computed tomography finding of cecal cancer (arrow) (A) and lymph node in portocaval space (arrow) (B).

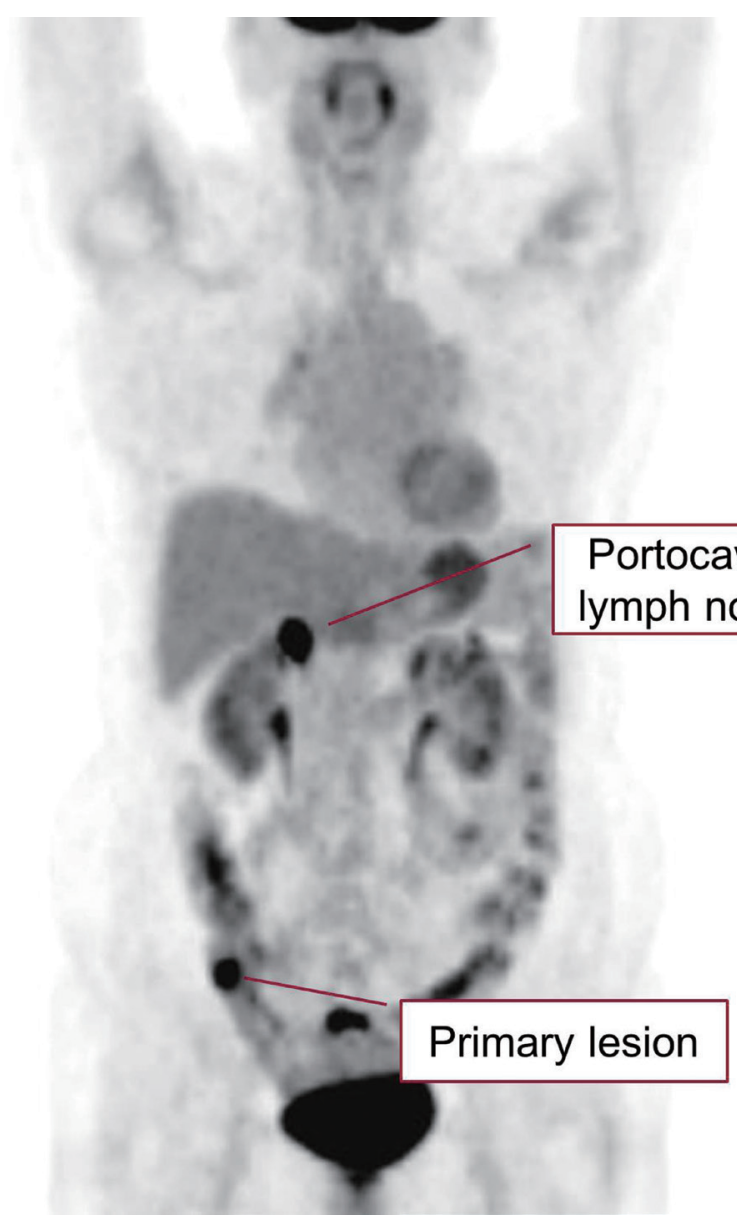

Fig. 3. Positron emission tomography-computed tomography finding of increased fluorodeoxyglucose (FDG) uptake in cecum and portocaval lymph node.

tion. Abdominopelvic computed tomography scan revealed cecal cancer without pericolic infiltration, and enlarged necrotic lymph node in portocaval space (Fig. 2). Positron emission tomography-computed tomography (PET-CT) scan showed increased fluorodeoxyglucose (FDG) uptake of proven cecal cancer and enlarged lymph node in the portocaval space (Fig. 3).

Right hemicolectomy with extensive lymph node dissection including portocaval, retropancreatic, hepatoduodenal lymph node was carried out. The portocaval lymph node was quite hard and a grossly metastatic lymph node. The resected portocaval lymph node was sent to the pathology department for frozen-section diagnosis and the result was metastatic adenocarcinoma. Whole abdominal cavity exploration was done including remnant colon, entire small bowel, stomach, and all intraabdominal organs. However, there was no suspicious lesion indicative of metastatic lesion. She was discharged 8 postoperative days later without any complication. The final pathology revealed a poorly differentiated adenocarcinoma. The cancer invaded the submucosa and the focally su- 
perficial layer of the muscularis, which is pT2 according to the American Joint Committee on Cancer (AJCC) 7th edition. A total of 27 lymph nodes were obtained and two of them involved metastatic adenocarcinoma. One of the metastatic lymph nodes was from the portocaval lymph node, which was confirmed by frozen-section biopsy and the other lymph node was from retropancreatic lymph node. There was no metastatic adenocarcinoma found among pericolic, intermediate and principle lymph nodes. The cecal cancer had lymphovascular invasion but not perineural invasion. The patient was finally diagnosed with cecal cancer stage IVa (T2N0M1a) according to the AJCC 7th edition and was scheduled for adjuvant chemotherapy.

\section{DISCUSSION}

Lymphatic spread of colon cancer sequentially advances from submucosal lymphoid follicle to pericolic, intermediate and principle lymph node [5]. Therefore, lymph node dissection including principle lymph node is the standard extent for colon cancer [6]. However, in cases of early colon cancer, the extent of lymph node dissection can be minimized because the incidence of lymphatic spread is low. For clinical T1 colon cancer which invades to the submucosal layer, the incidence of lymph node metastasis is approximately $0 \%$ to $7 \%[1,2]$. For clinical T2 colon cancer which invades to the muscularis propria, the incidence of lymph node metastasis is $9 \%$ to $20 \%[2,3]$. Kobayashi et al. [7] reported that lymph node dissection only to regional lymph nodes is sufficient in cT1N0 colon cancer. Kotake et al. [8] reported that lymph node dissection including regional lymph nodes and intermediate lymph nodes is sufficient in cT1N+and cT2N0 colon cancer [7].

However, distant lymph node metastasis without regional lymph node metastasis rarely occurs and it is called "skip metastasis" [4,5]. The incidence of skip metastasis is $1 \%$ to 3\% [9]. Many skip metastases were metastasis to principle lymph node without regional lymph node metastasis. Skip metastasis to distant lymph nodes has rarely been reported. There was a case report of skip metastasis to the external iliac and inguinal lymph node metastasis without regional lymph node metastasis [10]. In cases of cecal cancer, regional lymph nodes and lymph nodes around the ileocolic artery can metastasize preferentially. However, this is a very rare case of skip metastasis to the portocaval and retropancreatic space without regional lymph node metastasis.

During operation, portocaval lymph node was diagnosed as metastatic adenocarcinoma through frozen-section examination. Thorough exploration of the abdominal cavity was done to discover other metastatic lesions. However, there was no evidence of any other primary or metastatic cancer. This metastatic portocaval lymph node can be considered as carcinoma of unknown primary cancer (CUP). The most common type of CUP is known as adenocarcinoma, and possible primary sites are the lung, pancreas, colon, stomach, breast, ovary, and prostate [11]. However, final immunohistochemical stain revealed metastatic adenocarcinoma from colorectal origin in this case.

The colonoscopic finding of this case was considered as early cecal cancer due to its gross appearance. Despite clinically early cancer, detailed and sufficient preoperative evaluation helped detect skip metastasis. PET-CT scan demonstrated enlarged lymph node in the portocaval space and increased FDG uptake. Preoperative evaluation led us to perform radical surgery including portocaval and retropancreatic space dissection, which was essential for R0 resection.

In this report, skip metastasis could have been missed because of clinically early stage and rarity. Furthermore, it suggests that extensive lymph node dissection should be done if distant lymph node metastasis is suggested. Laparoscopic surgery is commonly performed, especially in early colon cancer [12]. However, a limitation of laparoscopic surgery is difficulty in evaluation of the whole abdominal cavity. Therefore, detailed and sufficient preoperative evaluation for clinical and imaging finding is important not to ignore skip metastasis.

\section{CONFLICT OF INTEREST}

No potential conflict of interest relevant to this article was reported.

\section{REFERENCES}

1. Nivatvongs S, Rojanasakul A, Reiman HM, Dozois RR, Wolff BG, Pemberton JH, et al. The risk of lymph node metastasis in colorectal polyps with invasive adenocarcinoma. Dis Colon Rectum 1991; 34:323-8.

2. Morikawa E, Yasutomi M, Shindou K, Matsuda T, Mori N, Hida J, et al. Distribution of metastatic lymph nodes in colorectal cancer by the modified clearing method. Dis Colon Rectum 1994;37:21923.

3. Baxter NN, Garcia-Aguilar J. Organ preservation for rectal cancer. JClin Oncol 2007;25:1014-20.

4. Herrera-Ornelas L, Justiniano J, Castillo N, Petrelli NJ, Stulc JP, Mittelman A. Metastases in small lymph nodes from colon cancer. Arch Surg 1987;122:1253-6.

5. Shiozawa M, Akaike M, Yamada R, Godai T, Yamamoto N, Saito H, et al. Clinicopathological features of skip metastasis in colorectal cancer. Hepatogastroenterology 2007;54:81-4.

6. Hohenberger W, Weber K, Matzel K, Papadopoulos T, Merkel S. 
Standardized surgery for colonic cancer: complete mesocolic excision and central ligation-technical notes and outcome. Colorectal Dis 2009;11:354-64.

7. Kobayashi Y, Fujita S, Yamaguchi T, Yamamoto S, Akasu T, Moriya Y. Optimum lymph node dissection in clinical T1 and clinical T2 colorectal cancer. Dis Colon Rectum 2009;52:942-9.

8. Kotake K, Kobayashi H, Asano M, Ozawa H, Sugihara K. Influence of extent of lymph node dissection on survival for patients with pT2 colon cancer. Int J Colorectal Dis 2015;30:813-20.

9. Merrie AE, Phillips LV, Yun K, McCall JL. Skip metastases in colon cancer: assessment by lymph node mapping using molecular detection. Surgery 2001;129:684-91.
10. Kitano Y, Kuramoto M, Masuda T, Kuroda D, Yamamoto K, Ikeshima S, et al. Ascending colon cancer with synchronous external iliac and inguinal lymph node metastases but without regional lymph node metastasis: a case report and brief literature review. Surg Case Rep 2017;3:32.

11. Oien KA, Dennis JL. Diagnostic work-up of carcinoma of unknown primary: from immunohistochemistry to molecular profiling. Ann Oncol 2012;23 Suppl 10:x271-7.

12. Bardakcioglu O, Khan A, Aldridge C, Chen J. Growth of laparoscopic colectomy in the United States: analysis of regional and socioeconomic factors over time. Ann Surg 2013;258:270-4. 